scholarship. This expectation is enhanced by the fact that the author confines himself to an early period and that the present volume is a second edition of a valuable work that has been before the public for sixteen years. The typographical errors are few and insignificant. The most serious noticed occurs on page 4 , where it is stated that the earlier edition appeared in 1900, instead of 1890 .

In 1838 Liouville demonstrated a property of the special forms which were afterwards called wronskians by Muir (1881) and later writers. According to Anissimov* this seems to be the first important result relating to these useful forms, but the paper is not mentioned by our author although it falls within the period covered. Neither is it included in the bibliography cited above.

The present volume is almost twice as large as the first edition and includes a number of papers not there mentioned. The greatest difference between the two editions is in the facts that the papers relating to special forms are brought together in the new edition and that a table showing the advance of determinants from 1813 to 1841 has been added. The use of smaller type for quotations has greatly improved the appearance of the text. The completeness and attractiveness of the book combine to make it indispensable to the student of determinants and their history. G. A. Miller.

\title{
A First Course in Analytical Geometry, Plane and Solid, with
} Numerous Examples. By Charles N. Schmall. New York, Van Nostrand, 1905. 8 vo. $7+318$ pp.

ON the whole, this little book resembles so closely the ordinary text on the subject, both as to material and treatment, that a detailed account is scarcely necessary. Mention of the few points in which it does depart from the general type of text will be sufficient. In the chapter on loci, the usual examples from physics, economics, statistics, etc., are omitted, and attention is confined exclusively to purely geometric problems. This omission will probably be regarded as a fault or a merit according to one's views on the recently discussed relations of mathematics to physics and other subjects. The chapter on the circle precedes that on the transformation of coordinates, reversing p. 3.

* Enoyclopédie des Sciences Mathématiques, Tribune publique I, 1906, 
the usual order. A short chapter on confocal conics is inserted, while the treatment of higher plane curves and of solid geometry are unusually brief.

The most interesting novel feature is the early introduction of the determinant notation and its continued use throughout the book. In the first equation of the straight line (i. e., in terms of the coordinates of two known points) the determinant form is given side by side with the explicit equation. Even if the student has had no previous treatment of the determinant, a few minutes of class-room explanation will enable him to grasp this simple form and the continued use will certainly give him that realizing sense of the geometric value of this notation which he too often fails to get in his formal course in algebra.

E. B. Cowley.

Lehrbuch der analytischen Geometrie. Erster Band: Geometrie in den Grundgebilden erster Stufe und in der Ebene. Von L. Heffter und C. Koehler. Leipzig, Teubner, 1905. 8vo. $16+526 \mathrm{pp}$.

THIS volume is in strong contrast to the book just noticed. While the authors aim to make the treatment elementary, they wish to give the student an introduction, at least, to the modern methods of relating the various parts and kinds of geometry into one comprehensive whole. They endeavor to follow the way suggested by Cayley's Sixth memoir on quantics and sketched by Klein in his Erlangen Programm. They consider that this can be done only by the early introduction of the transformation group, proceeding from the projective group to its subgroup, the affine, and then to its subgroup, the so-called "äquiform"; and not in the inverse order. This first volume contains the geometry in all spaces of one dimension, and in the plane. The second will be devoted to the finite "Bündel" and to ordinary space. In each case, the procedure is from projective to affine and then to æquiform geometry.

A condensed account of the contents will be useful in giving some idea of the ground covered. After an introductory chapter devoted to definitions and a few general considerations, there follows the first part, which is on geometry in spaces of one dimension. The first chapter relates to projective and affine geometry in the point range. The next takes up the quadratic equation and the point pair and its involution. This part is concluded by a consideration of the projective and 